\title{
A Concise Fuzzy Rule Base to Reason Student Performance Based on Rough-Fuzzy Approach
}

\author{
Norazah Yusof, Nor Bahiah Ahmad, \\ Mohd. Shahizan Othman and Yeap Chun Nyen \\ Faculty of Computer Science and Information System, \\ Universiti Teknologi Malaysia, Skudai, Johor \\ Malaysia
}

\section{Introduction}

A fuzzy inference system employing fuzzy if then rules able to model the qualitative aspects of human expertise and reasoning processes without employing precise quantitative analyses. This is due to the fact that the problem in acquiring knowledge from human experts is that much of the information is uncertain, inconsistent, vague and incomplete (Khoo and Zhai, 2001; Tsaganou et al., 2002; San Pedro and Burstein, 2003; Yang et al., 2005). The drawbacks of FIS are that a lot of trial and error effort need to be taken into account in order to define the best fitted membership functions (Taylan and Karagözoğlu, 2009) and no standard methods exist for transforming human knowledge or experience into the rule base (Jang, 1993).

Evaluation and reasoning of student's learning achievement is the process of determining the performance levels of individual students in relation to educational objectives (Saleh and Kim, 2009). Although Fuzzy inference system is a potential technique to reason the student's performance, as well as to present his/her knowledge status (Nedic et al., 2002; Xu et al., 2002; Kosba et al. 2003), it is a challenge when more than one factor involve in determining the student's performance or knowledge status (Yusof et. al, 2009). Hence, the reasoning of the student's performance for multiple factors is difficult. This issue is critical considering that the human experts' knowledge is insufficient to analyze all possible conditions as the information gained is always incomplete, inconsistent, and vague.

Addressing these matters, this work proposes a Neuro-Fuzzy Inference System (ANFIS), which combines fuzzy inference system and neural network, in order to produce a complete fuzzy rule base system (Jang, 1993). The fuzzy system represents knowledge in an interpretable manner, while the neural networks have the learning ability platform to optimize its parameters. Hence, ANFIS has the capability to perform parameter-learning rather than structural learning (Lin and Lu, 1996). ANFIS is expected to recognize other decisions that are previously not complete, in both the antecedents and consequent parts of the fuzzy rules. Unfortunately, too many fuzzy rules will result in a large computation time and space (Jamshidi, 2001). Therefore, reduction of knowledge is possible to be applied to determine the selection of important attributes that can be used to represent the decision system (Chen, 1999) based on the theory of rough sets. Fig. 1 shows the proposed fuzzy inference system. 


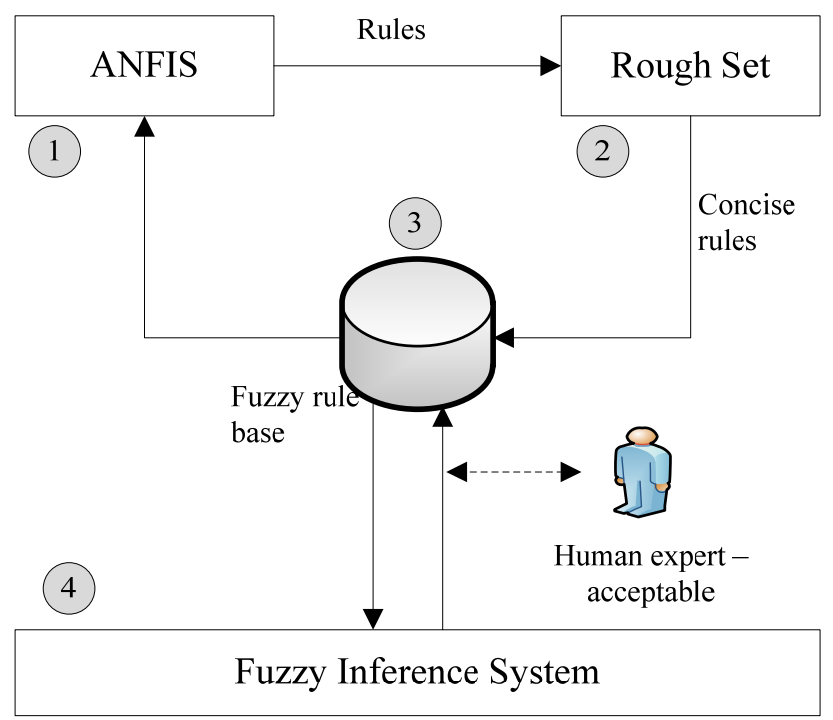

Fig. 1. The proposed Fuzzy Inference System

This chapter is divided into six sections. Section 1 is the introduction and the problem statements. Section 2 discusses about the student modeling and learning criteria. Section 3 presents the Human Expert Fuzzy Inference System model that defines the data representation and the rule base acquired from the human experts. Section 4 describes the ANFIS approach to form a complete fuzzy rule base to solve the problem of incomplete and vague decisions made by human. Section 5 presents the proposed Rough-Fuzzy approach to determine important attributes and refine the fuzzy rule base into a concise fuzzy rule base. Finally, section 6 presents the conclusions of the work.

\section{Student modeling and the learning criteria}

Student model represents the knowledge about the student's behavior and learning performance. In this work, student's performance are classified into three categories, named as Has Mastered (HM), Moderately Mastered (MM), and Not Mastered (NM). The conditions that determine the decision made about the student's performance is also depend on the criteria set by the human expert. There are four input conditions namely, the score $(S)$, time $(T)$, attempts $(A)$, and helps $(H)$ in which each of the input condition is represented by three term sets with values (Norazah, 2005).

a. Score (S) is the average scoring, $x_{1}$, which gains from each question of a learning unit and the term sets is represented by low $\left(S_{1}\right)$, moderate $\left(S_{2}\right)$, and high $\left(S_{3}\right)$. It can be found by dividing the total marks for a set of given questions by the total number of questions $(Q)$ in the set, as shown in equation (1).

$$
x_{1}=\frac{\sum_{i=1}^{Q} m_{i}}{Q}
$$


Where :

$m_{i}$ is marks from each question

$Q$ is total number the question in the set

b. Time $(\mathrm{T})$ is the average duration, $x_{2}$, taken by a student to answer the each question of a learning unit and with three term sets: fast $\left(T_{1}\right)$, average $\left(T_{2}\right)$, and slow $\left(T_{3}\right)$. The average of time $\left(x_{2}\right)$ is obtained by dividing the total time to answer a set of given questions by the total number of questions, see equation (2).

$$
x_{2}=\frac{\sum_{i=1}^{Q} T_{i}}{Q}
$$

Where :

$Q$ is total number of questions

$T_{i}$ is the time spent to answer the $i$-th question

Measurement of time can be done by using the distribution method. Fig. 2 shows the T-score distribution, in which the mean is 50 and the standard deviation is 10 .

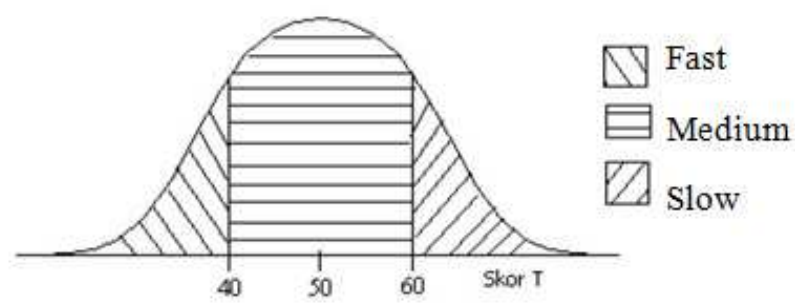

Fig. 2. T-score distribution for time taken to answer question

The time taken to answer each question $\left(T_{i}\right)$ can be calculated by using the equation (3).

$$
T_{i}=\frac{\frac{10\left(X_{i}-\bar{X}_{i}\right)}{\sigma_{i}}+50}{100}
$$

Where :

$T_{i}$ is the time spent to answer the $i$-th question

$X_{i}$ is the time spent by the student

$\bar{X}_{i}$ is mean score for the time spent distribution

$\sigma_{i}$ is the standard deviation for the $i$-th question

The numbered "10" is distance value of standard deviation from mean, while numbered "50" is value of mean. T-score is divided by 100 so that able to get the value in the range 0 to 1 .

c. Attempt $(\mathrm{A})$ is the average number of tries, $x_{3}$, for a given learning unit, in which it is counted after student give a wrong answer for the first attempt and the question will repeat again for student to answer until correct. The term sets involve: a few $\left(A_{1}\right)$, average $\left(A_{2}\right)$, and many $\left(A_{3}\right)$. The average of attempt $\left(x_{3}\right)$ is calculated as equation (4). Dividing the total number of tries to answer a set of given questions by the total number of questions in the set. 


$$
x_{3}=\frac{\sum_{i=1}^{Q} t_{i}}{Q}
$$

Where :

$Q$ is total number of questions

The number of attempt $\left(t_{i}\right)$ is determined by calculating the number of attempts made $\left(a_{i}\right)$ to answer a given question and dividing it by the maximum number of attempts $\left(P_{i}\right)$ allowed for the question.

$$
t_{i}=\frac{a_{i}}{P_{i}}
$$

Where :

$a_{i}$ is the number of attempts made to answer a given question

$P_{i}$ is the maximum number of attempts allowed for the question

d. Help $(\mathrm{H})$ is the average amount of help, $x_{4}$, of a learning unit where it able to help student by giving some hints or notes to answer the question. The term sets involve: little $\left(H_{1}\right)$, average $\left(H_{2}\right)$, and needed $\left(H_{3}\right)$.

The average amount of help $\left(x_{4}\right)$ is calculated as equation (6), by dividing the total amount of help accessed by a student in answering a set of given questions by the total number of questions in the set.

$$
x_{4}=\frac{\sum_{i=1}^{Q} h_{i}}{Q}
$$

Where :

$Q$ is the total number of questions

$h_{i}$ is the total amount of help accessed by a student

The amount of help $\left(h_{i}\right)$ is found by calculating the number of help $\left(l_{i}\right)$ links that a student accessed while answering a given question and dividing it by the maximum number of help links $\left(H_{\max }\right)$ provided for a given question.

$$
h_{i}=\frac{l_{i}}{H_{\max }}
$$

The output consequent of the student model is the student's performance and can be represented as has mastered $\left(P_{1}\right)$, moderately mastered $\left(P_{2}\right)$ and not mastered $\left(P_{3}\right)$ for the output. A student is classified as has mastered in a particular learning unit, when the student earns high scores (i.e. greater than $75 \%$ ) with below $40 \%$ of time spent, not exceeding $25 \%$ of number of tries needed and number of helps. Besides that, a student is classified as moderately mastered when the student earns a moderate score, with moderate time spent, tries more than once, and number of help needed. For example, a moderate score would be rated in between $35 \%$ and $75 \%$, time spent between $40 \%$ and $60 \%$, tries between $25 \%$ and $75 \%$, and help between $25 \%$ and $75 \%$. Furthermore, a student is classified as not mastered when the student has a low score with a lot of time, many tries and many help needed. However, in acquiring knowledge from the human experts is that, they cannot decide on all 
possible students learning performance. Bases on a survey done by Norazah (2005), there are only 18 decisions about the student's behavior are formed with certainty from seven subject matter experts; and these decisions are considered as the acceptable rules. All other decisions that are not certain and have conflicts are being discarded from the rules.

\begin{tabular}{ccccccc}
\hline Criteria item & \multicolumn{2}{c}{ Has Mastered } & \multicolumn{2}{c}{ Moderately Mastered } & \multicolumn{2}{c}{ Not Mastered } \\
& \multicolumn{2}{c}{$\mathbf{y}>\mathbf{7 5}$} & \multicolumn{7}{c}{$\mathbf{7 5} \geq \mathbf{y} \geq \mathbf{2 5}$} & \multicolumn{2}{c}{$\mathbf{2 5}$} \\
\cline { 2 - 7 } & Value & Label & Value & Label & Value & Label \\
\hline Scores $(S)$ & $x_{1} \geq 75 \%$ & High & $75 \% \geq x_{1} \geq 35 \%$ & Md & $x_{1}<35 \%$ & Low \\
Time $(T)$ & $x_{2}<40$ & Fast & $60 \geq x_{2} \geq 40$ & Avg & $x_{2}>60$ & Slow \\
Attempt $(A)$ & $x_{3}<25 \%$ & A Few & $75 \% \geq x_{3} \geq 25 \%$ & Avg & $x_{3}>75 \%$ & Many \\
Help $(H)$ & $x_{4}<25 \%$ & Little & $75 \% \geq x_{4} \geq 25 \%$ & Avg & $x_{4}>75 \%$ & Needed \\
\hline
\end{tabular}

Table 1. The criteria for the student's performance

\section{Human expert Fuzzy Inference System}

Human expert's FIS uses a collection of fuzzy membership functions and rules to reason about student's performance. FIS consists of a fuzzification interface, a rule base, a database, a decision-making unit, and finally a defuzzification interface.

To compute the output of this fuzzy inference system given the inputs, four steps has to be followed (Norazah, 2005):

a. Compare the input variables with the membership functions on the antecedent part to obtain the membership values of each linguistic label. This step is called fuzzification.

b. Combine the membership values on the premise part to get firing strength of each rule.

c. Generate the qualified consequents or each rule depending on the firing strength.

d. Aggregate the qualified consequents to produce a crisp output. This step is called defuzzification.

\subsection{Fuzzification}

In the fuzzification stage, the input and output of the fuzzy inference system are determined. Table 2 and Table 3 exhibit examples of the four input and one output

\begin{tabular}{ccc}
\hline Fuzzy input variable & Fuzzy linguistic terms & Numerical range (normalized) \\
\hline Score $(\mathrm{S})$ & \{Low, & {$[0.14,0.0]$} \\
& Moderate, & {$[0.12,0.55]$} \\
Time $(\mathrm{T})$ & High\} & {$[0.14,1.0]$} \\
& \{Fast, & {$[0.15,0.0]$} \\
& Average, & {$[0.08,0.5]$} \\
Attempt $(\mathrm{A})$ & Slow\} & {$[0.15,1.0]$} \\
& A f few, & {$[0.12,0.0]$} \\
& Average, & {$[0.12,0.5]$} \\
Help $(\mathrm{H})$ & Many\} & {$[0.12,1.0]$} \\
& ALittle, & {$[0.12,0.0]$} \\
& Average, & {$[0.12,0.5]$} \\
& Needed & {$[0.12,1.0]$} \\
\hline
\end{tabular}

Table 2. The input variables of the Fuzzy Inference System 
variables, in which each of the variables consists of three term values and labels as discussed in Section 2. The fuzzy output follows the zero-order Sugeno style inference, in which the output value of each fuzzy rule is a constant (Sivanandam et al., 2007). Fig. 3 shows the four inputs and one single output for the Human Expert FIS.

\begin{tabular}{ccc}
\hline Fuzzy output variable & Fuzzy linguistic terms & Numerical range(normalized) \\
\hline Performance $(\mathrm{P})$ & \{Not Mastered, & 0.0 \\
& Moderately Mastered, & 0.5 \\
& Has Mastered & 1.0 \\
\hline
\end{tabular}

Table 3. The output variables of the Fuzzy Inference System

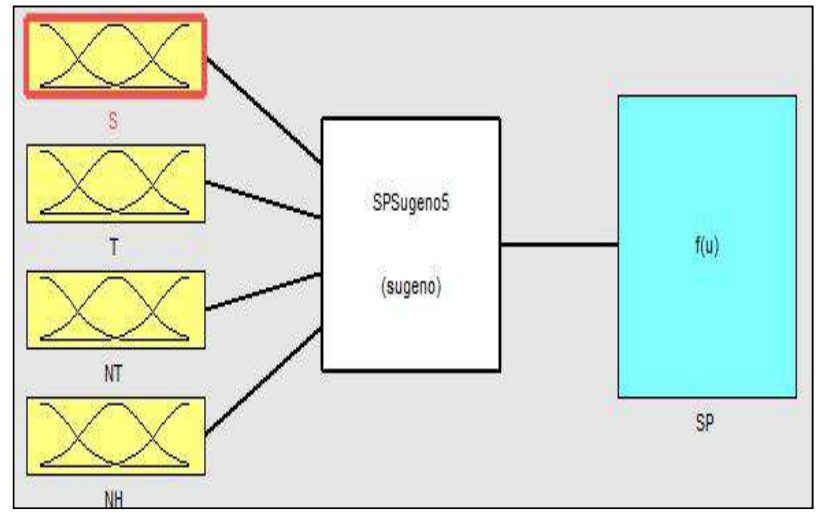

Fig. 3. Four inputs and single output for the Human Expert FIS

The membership function of the input is expressed by a Gaussian function specified by two parameters $\{\sigma, c\}$, and the membership value is derived by the formula in Fig. 4 .

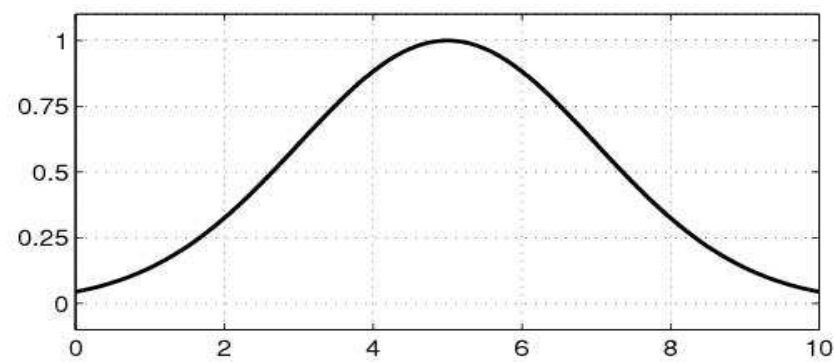

Fig. 4. Gaussian shape function $\operatorname{gaussian}(x ; 2,5)$

$$
\operatorname{gaussian}(x ; \sigma, c)=\exp \left(-\left[\frac{x-c}{2 \sigma}\right]^{2}\right)
$$

Where :

$c$ represents the membership function's center $\sigma$ determines the membership function's width 


\subsection{Creating fuzzy rules}

Fuzzy rules are a collection of linguistic statements that describe how the fuzzy inference system should make a decision regarding classifying an input or controlling an output. Fig. 5 presents the four inputs and one output reasoning of the student's performance procedure for zero order Sugeno fuzzy model. Each input has its own membership function.

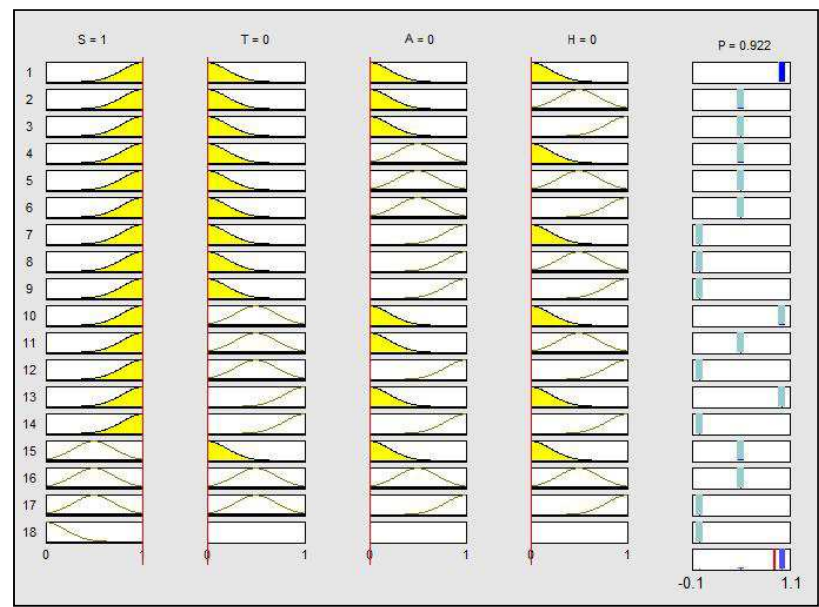

Fig. 5. Fuzzy reasoning procedures for Human Expert FIS model of Student's Performance

$R_{\mathrm{i}}$ have four input variables and one output variable as shown below:

$R_{i}$ : IF $S$ is $\mu_{i 1}$ AND $T$ is $\mu_{i 2}$ AND $A$ is $\mu_{i 3}$ AND $H$ is $\mu_{i 4}$ THEN $P$ is $w_{i}$

The rule $R_{i}$ is the $i$-th rule in the fuzzy rule base system, the $\mu_{i}$ is the membership function of the antecedent part of the $i$-th rule for each input variable and $w_{i}$ is the weight of the consequent of each rule. For example, for input1 is score and the membership function can classified as low, moderate or high. If score is high and time is fast and attempt is a few and help is little then student performance is has mastered. This process of taking input such as score and processing it through membership functions to determine the "high" score is called fuzzification. Based on the human experts' experience and knowledge about the students' performance, 18 initial rules that are certain have been constructed as shown in Table 4.

\subsection{Combining outputs into an output distribution}

The outputs of all of the fuzzy rules must now be combined to obtain one fuzzy output distribution. The output membership functions on the right-hand side of the figure are combined using the fuzzy operator AND to obtain the output distribution shown on the lower right corner of the Fig. 5. For a zero-order Sugeno model, the output level $z$ is a constant. The output level $z_{i}$ of each rule is weighted by the firing strength $w_{i}$ of the rule (Lin and $\mathrm{Lu}, 1996)$. For example, for an $\cap$ rule with input $1=x$ and input $2=y$, the firing strength is as shown in equation (9).

$$
w_{i}=F_{1}(x) \cap F_{2}(y)
$$


Where:

$F_{1}$ and $F_{2}$ are the membership functions for input 1 and 2, respectively

\begin{tabular}{cccccc}
\hline Rule & S & T & A & H & P \\
\hline 1 & High & Fast & A few & Little & Has Mastered \\
2 & High & Fast & A few & Average & Moderately Mastered \\
3 & High & Fast & A few & Needed & Moderately Mastered \\
4 & High & Fast & Average & Little & Moderately Mastered \\
5 & High & Fast & Average & Average & Moderately Mastered \\
6 & High & Fast & Average & Needed & Moderately Mastered \\
7 & High & Fast & Many & Little & Not Mastered \\
8 & High & Fast & Many & Average & Not Mastered \\
9 & High & Fast & Many & Needed & Not Mastered \\
10 & High & Average & A few & Little & Has Mastered \\
11 & High & Average & A few & Average & Moderately Mastered \\
12 & High & Average & Many & Needed & Not Mastered \\
13 & High & Slow & A few & Little & Has Mastered \\
14 & High & Slow & Many & Needed & Not Mastered \\
15 & Moderate & Fast & A few & Little & Moderately Mastered \\
16 & Moderate & Average & Average & Average & Moderately Mastered \\
17 & Moderate & Average & Many & Needed & Not Mastered \\
18 & Low & $x$ & $x$ & $x$ & Not Mastered \\
\hline
\end{tabular}

Table 4. Initial fuzzy rules determine by human experts

\subsection{Defuzzification of output distribution}

The input for the defuzzification process is a fuzzy set and the output is a single number crispness recovered from fuzziness. Given a fuzzy set that encompasses a range of output values, we need to return one number, thereby moving from a fuzzy set to a crisp output. The final output of the system is the weighted average of all rule outputs, computed as in equation (10).

$$
\text { Final Output }=\frac{\sum_{i=1}^{N} w_{i} z_{i}}{\sum_{i=1}^{N} w_{i}}
$$

Finally, all the outputs of datasets for reasoning of the student's performance in the human expert FIS have been recorded.

Next section describes the ANFIS approach to form a complete fuzzy rule base to solve the problem of incomplete and vague decisions made by human.

\section{Development of Adaptive Neuro-Fuzzy Inference System (ANFIS)}

Basically, fuzzy rules and fuzzy reasoning are the backbone of fuzzy inference systems, which are the most important modeling tools based on fuzzy sets (Jang et al., 1997). Fuzzy reasoning is an inference procedure that derives conclusions from the set of fuzzy If-Then 
rules and known facts. The ANFIS model is proposed to form a complete fuzzy rule bases so that all possible input conditions of the fuzzy rules are being generated.

It is necessary to take into consideration the scarcity of data and the style of input space partitions. For example, for a single input problem, usually 10 data points are necessary to come up with a good model (Jang et al., 1997). Details on ANFIS model structure will be described in section 4.1.

\subsection{ANFIS model structure}

The ANFIS model structure consists of four nodes for input layer, the nodes of hidden layer and one node for output layer as presented in Fig. 6. The input layer represents the antecedent part of the fuzzy rule, which is the student's learning behavior such as the scores $(S)$ earned, the time $(T)$ spent, the attempts $(A)$, and helps $(H)$; the output layer represents the consequent part of the rule, i.e. the student's performance $(P)$. The size of the hidden layer is determined experimentally.

In this work, the ANFIS model is trained with 18 fuzzy rules obtained from the human expert. These rules are considered as the rules that are certain. After that, 81 potential fuzzy rules are used for testing the network that represent the $3 \times 3 \times 3 \times 3$ rule antecedents.

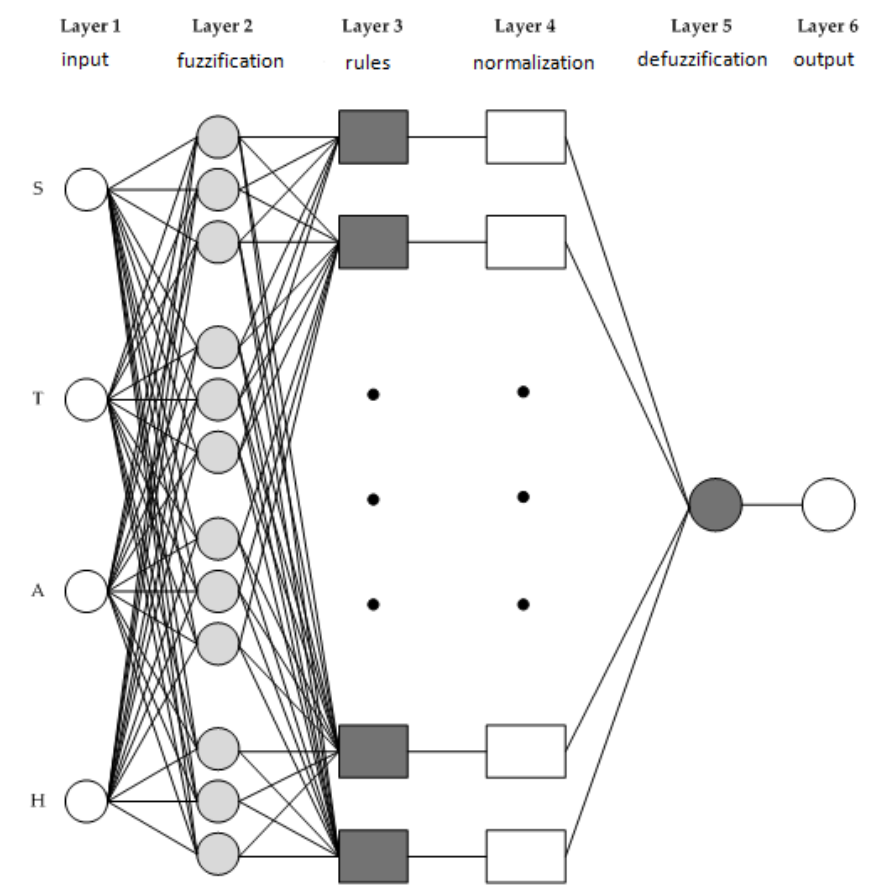

Fig. 6. ANFIS model structure

From the Fig. 6, every nodes of the same layer have similar functions. Layer 1 is the input layer and the neurons in this layer simply pass external crisp signals to Layer 2. 


$$
\begin{gathered}
y_{i}^{(1)}=\mu_{S i}(S) \\
y_{i}^{(1)}=\mu_{T i}(T) \\
y_{i}^{(1)}=\mu_{A i}(A) \\
y_{i}^{(1)}=\mu_{H i}(H)
\end{gathered}
$$

Where:

$\mu_{S i}(S), \mu_{T i}(T), \mu_{A i}(A), \mu_{H i}(H)$ are the input

$S_{i}, T_{i}, A_{i}, H_{i}$ are the linguistic value

$y_{i}^{(1)}$ is the output of input neuron $i$ in Layer 1

Layer 2 is the fuzzification layer. Neurons in this layer perform fuzzification. In this student model, fuzzification neurons have a Gaussian function. A Gaussian function, which has a Gaussian shape, is specified as:

$$
y_{i}^{(2)}=e^{-\left(\frac{x_{i}^{(2)}-c_{i}}{2 \sigma}\right)^{2}}
$$

Where:

$x_{i}^{(2)}$ is the input

$y_{i}^{(2)}$ is the output of neuron $i$ in Layer 2

$c$ represents the membership function's center

$\sigma$ determines the membership function's width

Layer 3 is the rule layer. Each neuron in this layer corresponds to a single to a single Sugeno type fuzzy rule. A rule neuron receives inputs from the respective fuzzification neurons and calculates the firing strength of the rule it represents. In an ANFIS, the conjunction of the rule antecedents is evaluated by the operator product (Negnevitsky, 2005). Each node output represents the firing strength of a rule. Thus, the output of neuron $i$ in Layer 3 is obtain as,

$$
y_{i}^{(3)}=\overline{w_{l}}=\mu_{S i}(S) \times \mu_{T i}(T) \times \mu_{A i}(A) \times \mu_{H i}(H) \quad i=1,2
$$

Layer 4 is the normalization layer. Each neuron in this layer receives inputs from all neurons in the rule layer and calculates the normalized firing strength of a given rule. The normalized firing strength is the ratio of the firing strength of a given rule to the sum of firing strengths of all rules. It represents the contribution of a given rule to the final result.

$$
y_{i}^{(4)}=\overline{w_{l}}=\frac{w_{i}}{w_{1}+w_{2}+w_{3}+w_{4}}
$$

Layer 5 is the defuzzification layer. Each neuron in this layer is connected to the respective normalization neuron and also receives initial input $\mathrm{S}, \mathrm{T}, \mathrm{A}$, and $\mathrm{H}$. A defuzzification neuron calculates the weighted consequent value of a given rule as,

$$
y_{i}^{(5)}=\bar{w}_{l} f_{i}=\bar{w}_{l}\left(a_{i} S+b_{i} T+c_{i} N T+d_{i} N H+e_{i}\right)
$$


Where:

$\bar{w}_{l}$ is the output of the Layer 4

$y_{i}^{(5)}$ is the output of defuzzification neuron $i$ in Layer 5

$\left\{a_{i}, b_{i}, c_{i}, d_{i}, e_{i}\right\}$ is a set of consequent parameter of rule $i$

Layer 6 is represented by a single summation neuron. This neuron calculates the sum of outputs of all defuzzification neurons and produces the overall ANFIS output (y).

$$
y_{i}^{(6)}=\text { overall output }=\sum_{i} \bar{w}_{\iota} f_{i}=\frac{\sum_{i} w_{i} f_{i}}{\sum_{i} w_{i}}
$$

\subsection{Training with different training datasets}

The preparation of the input patterns for training the ANFIS involves the conversion of the linguistic terms of the fuzzy rules into numeric values. Initially, there are 44 rules that are the certain and consistent rules, which are obtained from the human experts. The total number of input patterns for the training datasets is 44 rather than 18, because the ' $x$ ' symbol used in rule-18 in Table 4 should be represented by all possible linguistic terms for the respective antecedents.

The increments of the training datasets are very important until the ANFIS model had provided the best result and reasonably able to classify all of the student performance. Due to insufficient training data problem, the increments of 10 training patterns were proposed. Therefore, besides the 44 input patterns for training, this research also proposes 54, 64 and 69 trained ANFIS model.

In order to determine the best ANFIS model, ten tests had been carried out for each model and calculate their mean square errors (MSE). The error is the difference between the training data output value, and the output of the ANFIS corresponding to the same training data input value. The ANFIS model with the lowest mean square errors is being chosen for the next experiment.

\subsection{Results and discussion on ANFIS}

This section explains the testing results of the three ANFIS model selected from the trained fuzzy inference system. All the results had been tabulated in a line graphs to compare between ANFIS output based on 44,54, 64 and 69 training datasets, respectively and the testing data.

In this section, four ANFIS model selected from the previous experiment are selected to test the 81 input data patterns. All the results had been tabulated into a line graphs to compare between the ANFIS output. Fig. 7 shows the comparison between ANFIS outputs based on 44 training datasets and testing data. There are $69.14 \%$ of the input patterns which are classified successfully and $30.86 \%$ which are misclassified.

Besides that, Fig. 8 shows the comparison between ANFIS outputs based on 54 training datasets and testing data. From the graph, there are $85.19 \%$ were classified successfully and $14.81 \%$ were misclassified. Therefore, the increment of the training datasets need to be executed, so that able to achieve better result. 


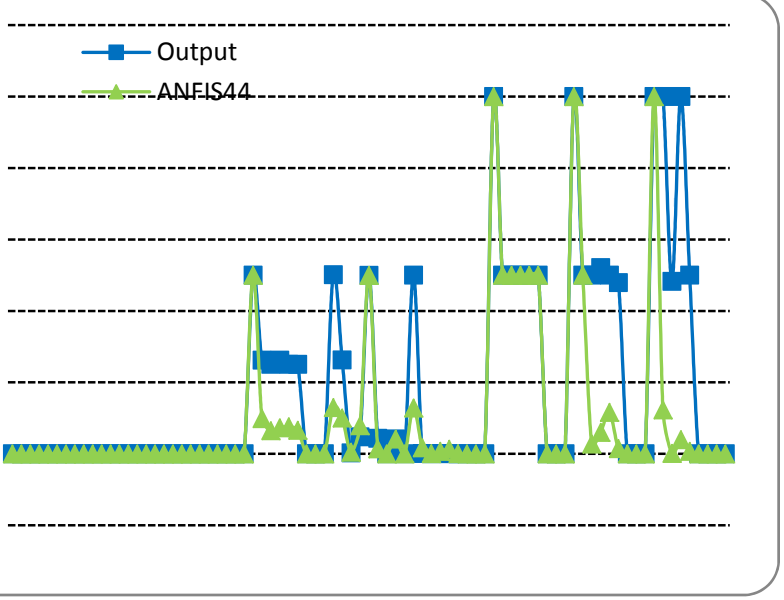

Fig. 7. Comparison between ANFIS outputs based on 44 training datasets and testing data

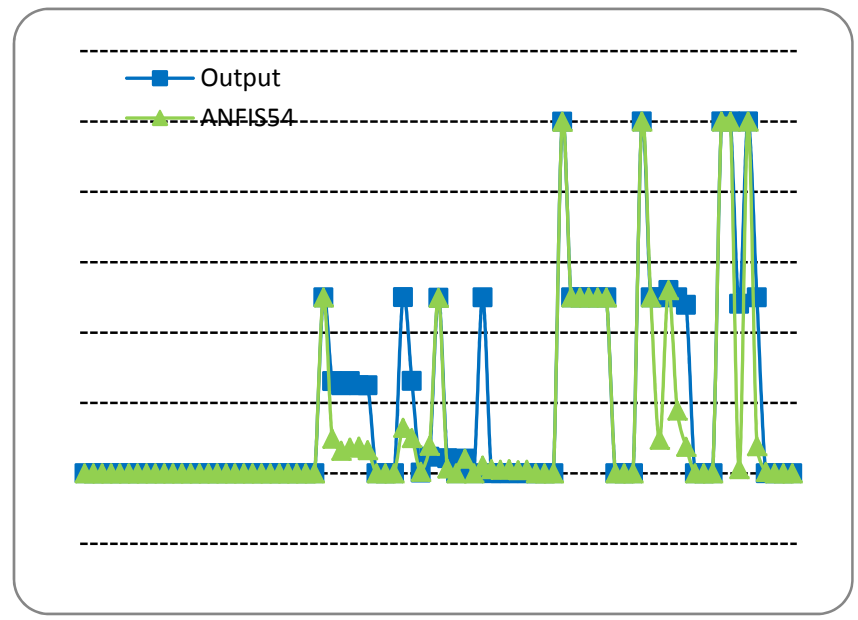

Fig. 8. Comparison between ANFIS outputs based on 54 training datasets and testing data

After incrementing the training data from 54 to 64, the results seem becomes better. Fig. 9 shows the comparison between outputs of ANFIS model based on 64 training datasets and outputs of the checking data. The outcomes of the trained ANFIS able to achieved up to $96.3 \%$ which are classified successfully. However, still have some of outputs are illogical decisions. There are $3.7 \%$ of the decisions are illogically.

Thus, another experiment carried out by using the 69 training datasets and finally the all the outputs of the ANFIS are able to classify all the 81 input patterns successfully. We can see it clearly in the Fig. 10. From the graph, both of the outputs are same and the ANFIS model can classify the student performance correctly in all possible conditions. 


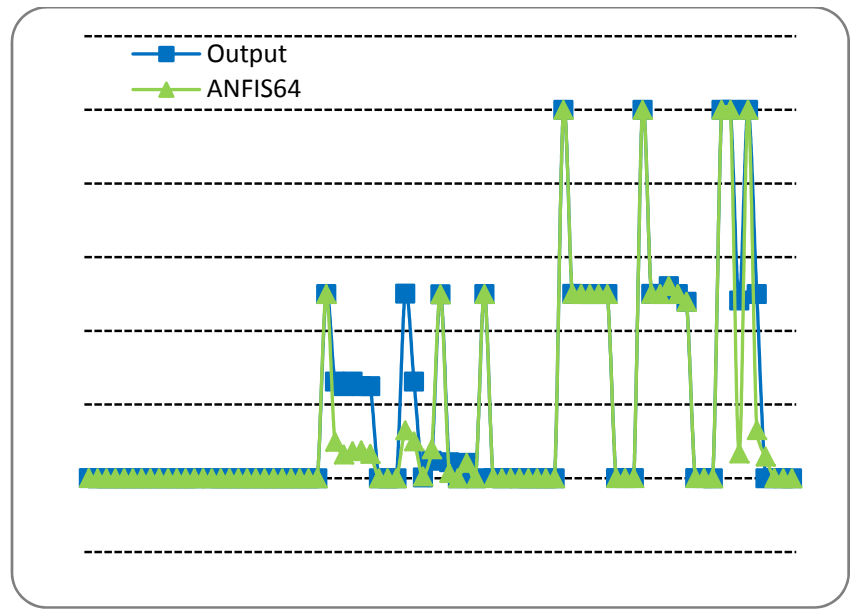

Fig. 9. Comparison between ANFIS outputs based on 64 training datasets and testing data

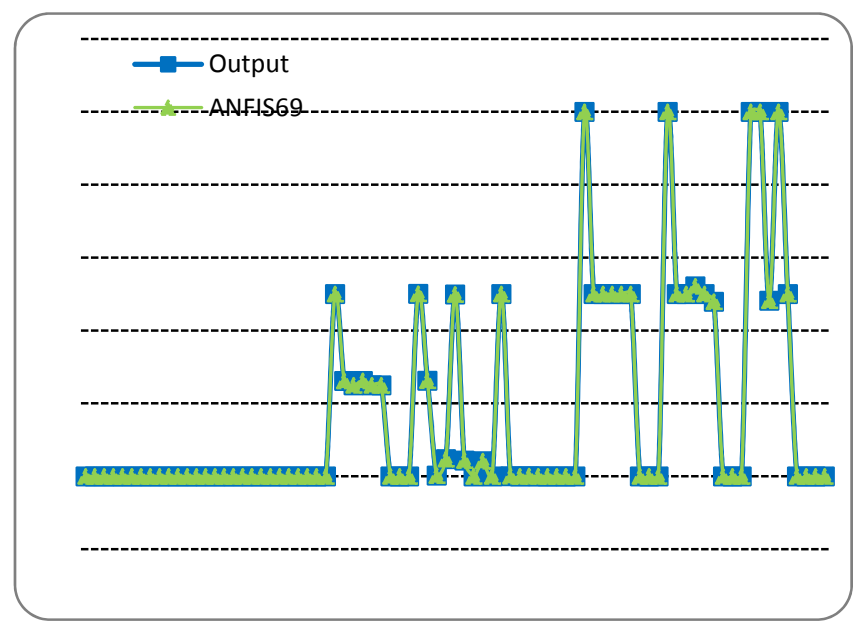

Fig. 10. Comparison between ANFIS outputs based on 69 training datasets and testing data

Moreover, the percentage of successful classification for each input data pattern have been calculated and shown in the Table 4 and Fig. 10. The table below indicates that the human experts' fuzzy rule base consisting of only 18 rules has the possibility of not giving all classification result. For 81 input datasets have been tested only $62 \%$ successfully classified; 1500 random input datasets, $66 \%$ successfully give the desired result. Meanwhile ANFIS based on 69 training datasets yield encouraging results than human experts' fuzzy rule base, they have successfully classified all the given input.

By analyzing and comparing the experimental results for the five fuzzy rule bases, it can be concluded that the human experts' fuzzy rule base is consistent but incomplete. This is 
because the 18 rules in this rule base were carefully selected to give full certainty for decisions. However, we found that not all situations covered by this 18 fuzzy rules and still have some rules are not stated. On the contrary, the complete fuzzy rule base in ANFIS is complete but still got some rules are inconsistent and the decision output is not logically. Although all situations for all four attributes are covered by the set of 81 rules, some of the rules have been found to have unnecessary conditions. Thus, the increment of the training data need to done, so that the ANFIS based on 69 training datasets able to eliminate the unnecessary conditions and the illogical decisions. Finally, the ANFIS model is consistent and complete; all situations for all four attributes are covered by the set of 69 training data, and there are no missing rules.

\begin{tabular}{lrr}
\hline \multirow{2}{*}{ Fuzzy Rule Base } & \multicolumn{2}{c}{ Input data patterns } \\
\cline { 2 - 3 } & $\mathbf{8 1}$ & $\mathbf{1 5 0 0}$ \\
\hline Human Experts & $62.00 \%$ & $66.00 \%$ \\
ANFIS (44) & $69.14 \%$ & $89.60 \%$ \\
ANFIS (54) & $85.19 \%$ & $99.47 \%$ \\
ANFIS (64) & $96.30 \%$ & $99.73 \%$ \\
ANFIS (69) & $100.00 \%$ & $100.00 \%$ \\
\hline
\end{tabular}

Table 5. Percentage of successful classifications correctly

\section{Rough-fuzzy approach}

ANFIS approach described in Section 4 has successfully formed a complete fuzzy rule that able to solve the problem of incomplete and vague decisions made by human. However, not all rules generated are significant and thus it is important to extract only the most significant rules in order to improve the classification accuracy. In this work, we propose Rough-Fuzzy approach to refine the fuzzy rule base into a concise fuzzy rule base (refer Fig. 11).

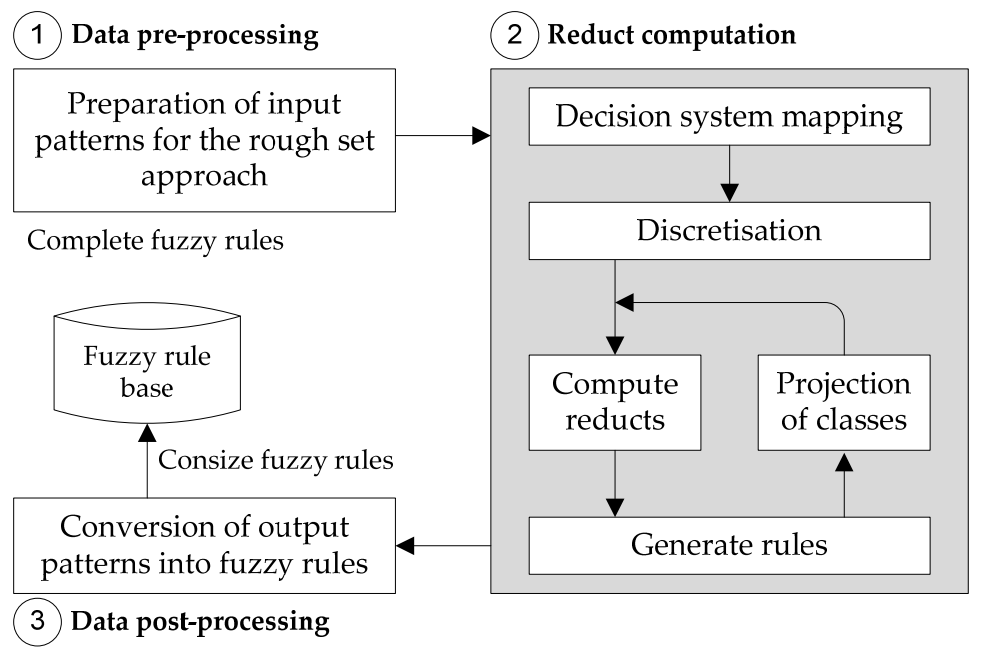

Fig. 11. The rough-fuzzy approach to constructing concise fuzzy rules 


\subsection{Rough fuzzy phases}

The three main phases in the rough-fuzzy approach are data pre-processing, reduct computation and data post-processing as shown in Fig. 11 and described as follows:

Phase 1. Data pre-processing.

In this phase, the complete fuzzy rules are converts from linguistic terms into numeric values that correspond to the rough set format.

Phase 2. Reduct computation.

The fuzzy rules are mapped into a decision system format, discretisation of data, computation of reducts from data and derivation of rules from reducts.

a. In this problem, the fuzzy rules are mapped as rows; while the antecedents and the consequents of the rules are mapped into columns. In the rough set decision table, the antecedents and consequents of the fuzzy rules are labelled as condition and decision attributes, respectively.

b. Discretisation refers to the process of arranging the attribute values into groups of similar values. It involves the transformation of the fuzzy linguistic descriptions of the conditions and the decision attributes into numerical values. In this study, a conversion scheme is formulated to transform the conditions and decisions of fuzzy linguistic values into numerical representations.

c. Computation of reduct

The reduct computation stage determines the selection of an important attribute that can be used to represent the decision system (Carlin et al., 1998). It is used to reduce the decision system, thus generating more concise rules. The rough set approach employs two important concepts related to reduction: one is related to reduction of rows, and the other one is related to reduction of columns (Chen, 1999). With the notion of an indiscernibility class, the rows with certain properties are grouped together, while with the notion of dispensable attributes, the columns with less important attributes are removed. Another essential concept in reduct computation is the lower and upper approximations, in which the computation involved in the lower approximation produces rules that are certain, while the computation involved in the upper approximation produces possible rules (Øhrn, 2001).

d. Rule Generation. A reduct is converted into a rule by binding the condition attribute values of the object class from which the reduct is originated to the corresponding attribute.

Phase 3. Data post-processing

The rules in rough set format are converted into linguistic terms of the concise fuzzy rule base.

\subsection{Rough fuzzy experiment}

In Section 4, there are 81 datasets that represent every possible value of the fuzzy rules with full certainty. This dataset is used for the development of the ANFIS model. Using Rosetta as rough set tool, the genetic algorithm with object reduct is the method used for computing reducts (Øhrn, 2001). This method implements a genetic algorithm for computing minimal hitting sets as described by Vinterbo and Øhrn (2000). Using rough set, we trained the fuzzy 
rules incrementally with different training data set that consist of 44, 54, 64 and 69 input data patterns as described in Section 4 . The purpose of the iteration with different input patterns of ANFIS is to ensure that the decision is agreed by human expert.

Table 6 shows the number of reducts, the number of rules and the rule percentage of rough set experiment with different input patterns. The result shows that ANFIS with 69 input patterns generates more concise rule with less number of reducts and less number of rules extracted compared to ANFIS with other pattern.

\begin{tabular}{llccl}
\hline Model & No of Reducts & No of Rules & Percentage of Rules \\
\hline 1. & Human expert & 6 & 13 & $16 \%$ \\
2. & ANFIS with 44 input patterns & 11 & 23 & $28 \%$ \\
3. & ANFIS with 54 input patterns & 9 & 16 & $20 \%$ \\
4. & ANFIS with 64 input patterns & 7 & 13 & $16 \%$ \\
5. & ANFIS with 69 input patterns & 4 & 8 & $10 \%$ \\
\hline
\end{tabular}

Table 6. Number of reducts and rules based on different input patterns

To determine whether the performance of the concise fuzzy rule base is consistent with the performance of the complete fuzzy rule base, each rule bases of input patterns is compared.

Table 7 shows that the decision output given by both the rule bases of each input patterns has very small differences (in terms of its mean square error). This result confirms that the concise fuzzy rule base does not degrade the performance of the complete fuzzy rule base.

It can be seen from Table 7 that ANFIS with 69 input patterns matched exactly as predicted by experts with MSE value equal to zero. The reducts and rules generated by rough set for ANFIS with 69 input patterns is chosen for further discussion.

\begin{tabular}{lrr}
\hline Complete Rule Base (81 Rules) & Concise Rule Base & \multicolumn{1}{l}{ MSE } \\
\hline ANFIS with 44 input patterns & 23 Rules & $4.76 \mathrm{E} 07$ \\
ANFIS with 54 input patterns & 16 Rules & $1.02 \mathrm{E} 07$ \\
ANFIS with 64 input patterns & 13 Rules & $3.70 \mathrm{E} 10$ \\
ANFIS with 69 input patterns & 8 Rules & 0.00 \\
\hline
\end{tabular}

Table 7. MSE result of Complete vs Concise Rule Base

Furthermore, Table 8 shows four object-related reduct generated by Rosetta for ANFIS with 69 input patterns. All reducts has $100 \%$ support, which mean that all objects are mapped deterministically into a decision class. In other words, the support for the decision rule is the probability of an object to be covered by the description that belongs to the class (GrzymalaBusse, 1991).

\begin{tabular}{lll}
\hline Class & Reduct & Support \\
\hline$C_{1}$ & $\{$ Score $\}$ & 100 \\
$C_{2}$ & $\{$ Attempt $\}$ & 100 \\
$C_{3}$ & $\{$ Score , Attempt $\}$ & 100 \\
$C_{4}$ & $\{$ Score, Attempt, Help $\}$ & 100 \\
\hline
\end{tabular}

Table 8. Object-related reduct based on ANFIS 69 model 
Rules generated from reduct are representative rules extracted from the data set. Since a reduct is not unique, rule sets generated from different reducts contain different sets of rules as shown in Table 9.

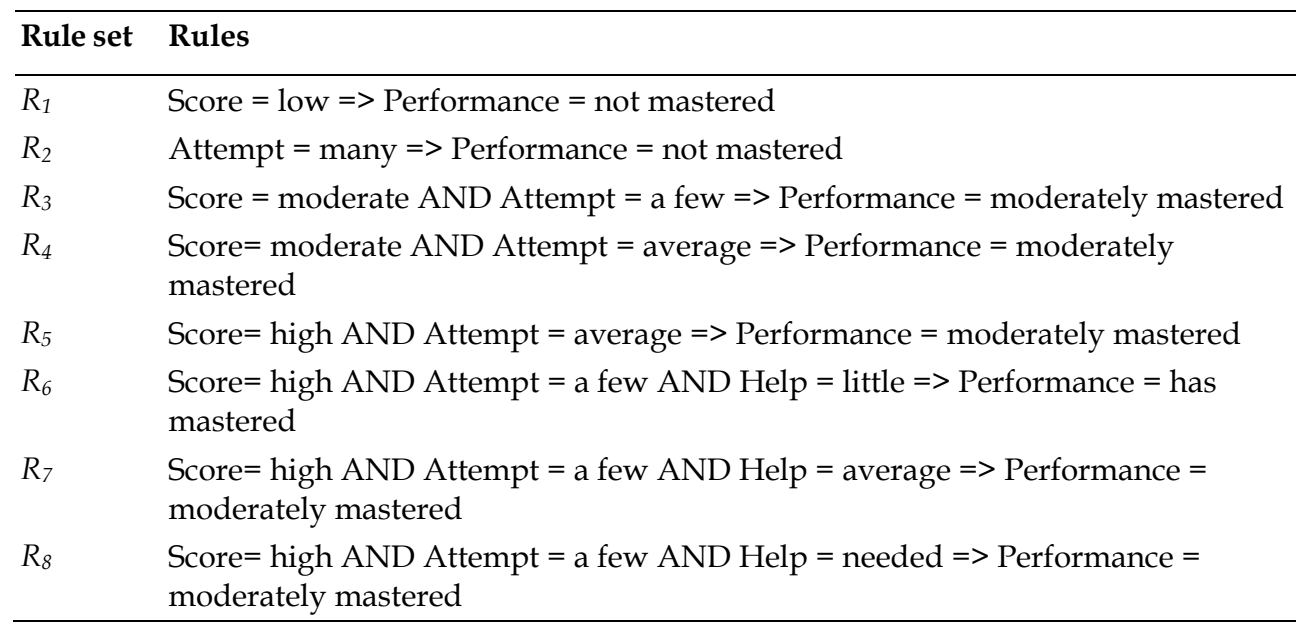

Table 9. Rule Generation

For example, the given reduct from Table 8 i.e. reduct $\{$ Score, Attempt\}, is presented by three rules as shown in Table 9 namely $R_{3}, R_{4}$, and $R_{5}$.

$R_{3}:$ IF Score $=$ moderate AND Attempt $=$ a few THEN Performance $=$ moderately mastered $R_{4}$ : IF Score $=$ moderate AND Attempt $=$ average THEN Performance $=$ moderately mastered

$R_{5}:$ IF Score $=$ high AND Attempt $=$ average THEN Performance $=$ moderately mastered

A unique feature of the rough set method is its generation of rules that played an important role in predicting the output. Table 10 listed the rule generation analysis by Rosetta and provides some statistics for the rules which are support, accuracy, coverage and length. The rule coverage and accuracy are measured to determine the reliability of the rules. Below is the definition of the rule statistics (Bose, 2006).

a. The rule support is defined as the number of records in the training data that fully exhibit property described by the IF condition.

b. The rule accuracy is defined as the number of RHS support divided by the number of LHS support.

c. The conditional coverage is the fraction of the records that satisfied the IF conditions of the rule. It is obtained by dividing the support of the rule by the total number of records in the training sample.

d. The decision coverage is the fraction of the training records that satisfied the THEN conditions. It is obtained by dividing the support of the rule by the number of records in the training that satisfied the THEN condition.

e. The rule length is defined as the number of conditional elements in the IF part. 


\begin{tabular}{llllll}
\hline RS & RSupp & RA & CA & DC & RL \\
\hline$R_{1}$ & 27 & 1 & $27 / 81=0.333333$ & $27 / 45=0.6$ & 1 \\
$R_{2}$ & 27 & 1 & $27 / 81=0.333333$ & $27 / 45=0.6$ & 1 \\
$R_{3}$ & 9 & 1 & $9 / 81=0.111111$ & $9 / 33=0.272727$ & 2 \\
$R_{4}$ & 9 & 1 & $9 / 81=0.111111$ & $9 / 33=0.272727$ & 2 \\
$R_{5}$ & 9 & 1 & $9 / 81=0.111111$ & $9 / 33=0.272727$ & 2 \\
$R_{6}$ & 3 & 1 & $3 / 81=0.037037$ & $3 / 3=1$ & 3 \\
$R_{7}$ & 3 & 1 & $3 / 81=0.037037$ & $3 / 33=0.090909$ & 3 \\
$R_{8}$ & 3 & 1 & $3 / 81=0.037037$ & $3 / 33=0.090909$ & 3 \\
\hline
\end{tabular}

Legend:

RS - Rule Sets, RSupp - Rule Support, RA - Rule Accuracy, CA - Conditional Coverage, DC - Decision Coverage, RL - Rule Length

Table 10. Rule Generation Analysis

Coverage gives a measure of how well the objects describe the decision class. The conditional coverage is measured by the ratio of the number of rules that fulfil the conditional part of the rules to the overall number of rules in the sample. Meanwhile, the decision coverage is measured by the ratio of the number of rules that give decision rules to the overall number of rules in the sample. Accuracy gives a measure of how trustworthy the rule is in the condition. It is the probability that an arbitrary object belonging to Class $C$ is covered by the description of the reduct (Grzymala-Busse, 1991). According to Pawlak (1998), an accuracy value of 1 indicates that the classes have been classified into decision classes with full certainty and consistency.

For example, there are 27 objects that fulfil the conditional part of the rule $R_{1}$, compared with the overall 81 rules in the sample. Therefore, the conditional coverage of this rule is about 0.3333 . In addition, the decision for the performance and learning efficiency with the value of not mastered is used once in the fuzzy rule base and it is only given to rule $R_{1}$. Therefore, the decision coverage for this rule is 1 . Finally, the accuracy value of this rule is 1 , which means that this rule belongs to Class $C_{1}$ and is covered. Thus, it is said to have full certainty and is consistent. In conclusion, because all of the rules in Table 10 have accuracy values of 1 , the concise fuzzy rules are reliable because they are covered, have full certainty, and are consistent.

\section{Conclusion}

In this study, fuzzy inference models provide an efficient way to reason about a student's learning achievement in quantitative way. In this work, a complete fuzzy rule base are formed using ANFIS approach, where all possible input conditions of the fuzzy rules are being generated apart from the 18 human experts' rules that are considered certain. By training the neural network with selected 18 conditions that are certain, the ANFIS is able to recognize other decisions that are previously not complete, in both the antecedents and consequent parts of the fuzzy rules. However, some of the decisions are found misclassified and inconsistent. In addition, it is realized that the number of fuzzy rules formed is directly related to the number of fuzzy term sets defined at the antecedents. As the number of fuzzy term sets increases, the fuzzy rules will also increases and will affect the computation time and space. Besides that, when there are too many rules, some of the rules may be found not 
significant. Therefore, this work proposes the Rough-Fuzzy approach that able to reduce the complete fuzzy rule base into a concise fuzzy rule base. This approach able to determine the selection of important attributes that can be used to represent the fuzzy rule base system. Therefore, the condition space is reduced by taking only a few conditions to achieve a reasonable size of the condition subspace. Moreover, the proposed concise fuzzy rule base is said to be reliable, due to the fact that it is covered, consistent and have full certainty.

\section{Acknowledgment}

The authors are especially grateful to the members of the Soft Computing Research Group (SCRG), Faculty of Computer Science and Information Systems, University of Technology Malaysia, for their encouraging support to this work. The authors would also like to thank Universiti Teknologi Malaysia (UTM) for their financial support under Research University Grant Vot. No. Q.J130000.7128.01H82 and Q.J130000.7128.02J57 as well as the FRGS Grant Vot No. 4F026(NT:2000957).

\section{References}

Bose, I. (2006). Deciding the Financial Health of Dotcoms using Rough Sets, Information $\mathcal{E}$ Management, Vol. 43, No. 7, pp. 835-846, October, ISSN 0378-7206.

Carlin, U.S.; Komorowski, J. \& Ohrn, A. (1998). Rough set analysis of patients with suspected acute appendicitis, Proceedings of Information Processing and Management of Uncertainty in Knowledge-based Systems, pp. 1528-1533.

Chen, Z. (1999). Computational Intelligence for Decision Support. CRC Press, ISBN 0849317991.

Grzymala-Busse, J.W. (1991). Managing Uncertainty in Expert Systems, Kluwer Academic Publishers, Boston, ISBN 0792391691.

Jamshidi, M. (2001). Autonomous control of complex systems: robotic applications, Applied Mathematics and Computation, Vol. 120, No. 1-3, pp. 15-29, ISSN 0096-3003.

Jang, J., -S. (1993). ANFIS:Adaptive-Network-Based Fuzzy Inference. IEEE Trans. on Systems, Man and Cybernetics 23: 665-685, ISSN 0018-9472.

Jang, J.-S. R.; Sun, C. -T., Mizutani, E. (1997). Neuro-Fuzzy And Soft Computing: A Computational Approach to Learning and Machine Intelligence. (1st ed.), United States of America: Prentice Hall, ISBN 0132610663.

Khoo, L.P. \& Zhai, L.Y. (2001). Rclass*: A Prototype Rough-set and Genetic Algorithms Enhanced Multi-concept Classification System for Manufacturing Diagnosis, CRC Press.

Kosba, E.; Dimitrova, V. \& Boyle, R., (2003) Using fuzzy techniques to model students in Web-based learning environment, in Palade, V., Howlett, R.J. \& Jain, L. (Eds.): Knowledge-based Intelligent Information and Engineering Systems, $7^{\text {th }}$ International Conference, KES 2003, Oxford, United Kingdom, September 2003, Proceedings Part II, pp. 222-229.

Lin, C. \& Lu, Y. (1996). A neural fuzzy system with fuzzy supervised learning, IEEE Transactions on Systems Man and Cybernetics, Part B 26, pp. 744-763, ISSN 1083-4419.

Nedic, Z.; Nedic, V. \& Machotka, J. (2002). Intelligent tutoring system for teaching 1st year engineering, World Transactions on Engineering and Technology Education 2002, UICEE, Vol. 1, No. 2, pp. 241-244.

Negnevitsky, M. (2005). Artificial Intelligence - A Guide to Intelligent Systems, Addison Wesley, ISBN 0-321-20466-2. 
Norazah, Y. (2005). Student Learning Assessment Model Using Hybrid Method. Phd thesis. Universiti Kebangsaan Malaysia, Malaysia.

Øhrn, A. (2001). Rosetta Technical Reference Manual, Department of Computer and Information Science, Norwegian University of Science and Technology, Trondheim, Norway.

Pawlak, Z. (1998). Reasoning about data - A rough set perspective, In: Polkowski, L. and Skowron, A. (eds.), Rough Sets and Current Trends in Computing '98, (pp. 25-34), Berlin: Springer-Verlag, ISBN 3-540-64655-8.

Saleh. I., \& Kim, S.I. (2009). A fuzzy system for evaluating student's learning achievement. Expert systems with Applications. 36(2009), pp. 6236-6243, ISSN 0957-4174.

San Pedro, J. \& Burstein, F. (2003). Intelligent assistance, retrieval, reminder and advice for fuzzy multicriteria decision-making, in Palade, V., Howlett, R.J. \& Jain, L.C. (Eds.): KES 2003, pp. 37-44, Springer-Verlag, Berlin, Heidelberg.

Sivanandam, S.N.; Sumathi, S., \& Deepa, S.N. (2007). Introduction to Fuzzy Logic using Matlab. (1st ed.) Berlin Heidelberg, New York: Springer, ISBN 3540357807.

Taylan, O. \& Karagözoğlu, B. (2009). An adaptive neurofuzzy model for prediction of student's academic performance. Computers $\mathcal{E}$ Industrial Engineering. pp. 1-10.

Tsaganou, G.; Grigoriadou, M. \& Cavoura, T. (2002). Modelling student's comprehension of historical text using fuzzy casebased reasoning, Proceedings of the 6th European Workshop on Case-based Reasoning for Education and Training, Aberdeen, Scotland.

Vinterbo, S. \& Øhrn A. (2000). Minimal approximate hitting sets and rule templates, International Journal of Approximate Reasoning, Vol. 25, No. 2, pp. 123-143, ISSN: 0888-613X.

$\mathrm{Xu}$, D.; Wang, H. \& Su, K. (2002). Intelligent student profiling with fuzzy models, Proceeding of the 35 th International Conference on System Sciences, pp. 1-8, ISBN:0-7695-1435-9.

Yang, Y.; Hinde, C. \& Gillingwater, D. (2005). A conceptual framework for society-oriented decision support, AI and Society, Vol. 19, pp.279-291, Springer-Verlag, ISSN 978-14244-5330-6.

Yusof, N.; Mohd. Zin, N. A., Mohd. Yassin, N., \& Samsuri P. (2009). Evaluation of Student's Performance and Learning Efficiency based on ANFIS, SocPar pp. 460 - 465, ISBN 978-1-4244-5330-6. 


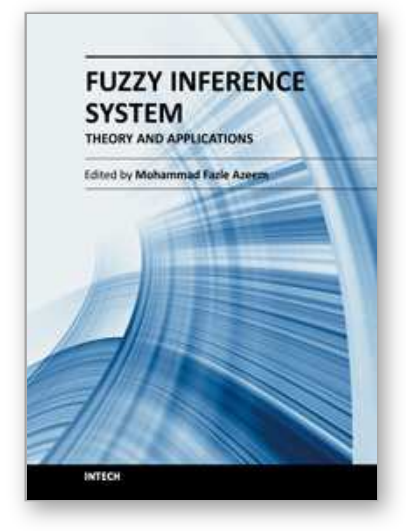

\author{
Fuzzy Inference System - Theory and Applications \\ Edited by Dr. Mohammad Fazle Azeem
}

ISBN 978-953-51-0525-1

Hard cover, 504 pages

Publisher InTech

Published online 09, May, 2012

Published in print edition May, 2012

This book is an attempt to accumulate the researches on diverse inter disciplinary field of engineering and management using Fuzzy Inference System (FIS). The book is organized in seven sections with twenty two chapters, covering a wide range of applications. Section I, caters theoretical aspects of FIS in chapter one. Section II, dealing with FIS applications to management related problems and consisting three chapters. Section III, accumulates six chapters to commemorate FIS application to mechanical and industrial engineering problems. Section IV, elaborates FIS application to image processing and cognition problems encompassing four chapters. Section V, describes FIS application to various power system engineering problem in three chapters. Section VI highlights the FIS application to system modeling and control problems and constitutes three chapters. Section VII accommodates two chapters and presents FIS application to civil engineering problem.

\title{
How to reference
}

In order to correctly reference this scholarly work, feel free to copy and paste the following:

Norazah Yusof, Nor Bahiah Ahmad, Mohd. Shahizan Othman and Yeap Chun Nyen (2012). A Concise Fuzzy Rule Base to Reason Student Performance Based on Rough-Fuzzy Approach, Fuzzy Inference System Theory and Applications, Dr. Mohammad Fazle Azeem (Ed.), ISBN: 978-953-51-0525-1, InTech, Available from: http://www.intechopen.com/books/fuzzy-inference-system-theory-and-applications/a-concise-fuzzy-rulebase-to-reason-student-performance-based-on-rough-fuzzy-approach

\section{INTECH}

open science | open minds

\section{InTech Europe}

University Campus STeP Ri

Slavka Krautzeka 83/A

51000 Rijeka, Croatia

Phone: +385 (51) 770447

Fax: +385 (51) 686166

www.intechopen.com

\section{InTech China}

Unit 405, Office Block, Hotel Equatorial Shanghai

No.65, Yan An Road (West), Shanghai, 200040, China

中国上海市延安西路65号上海国际贵都大饭店办公楼 405 单元

Phone: +86-21-62489820

Fax: $+86-21-62489821$ 
(C) 2012 The Author(s). Licensee IntechOpen. This is an open access article distributed under the terms of the Creative Commons Attribution 3.0 License, which permits unrestricted use, distribution, and reproduction in any medium, provided the original work is properly cited. 